\title{
Mujeres-madres que trabajan. La resignificación de la maternidad en mujeres profesionistas en Guadalajara-México
}

\section{Liliana Ibeth Castañeda-Rentería}

Universidad de Guadalajara

liliana.castaneda@cuci.udg.mx

\section{Karla Alejandra Contreras Tinoco}

Universidad de Guadalajara

ctka_28@hotmail.com

RESUMEN

Este trabajo tiene como objetivo el análisis de las resignificaciones sobre la maternidad en mujeres profesionistas con hijos residentes en Guadalajara, México. Sostenemos que las transformaciones en la representación sobre la maternidad y la madre - como instituciones socioculturales - son promovidas y sufridas por aquellas mujeres que «quieren todo», es decir, aquellas que no renuncian a su proyecto profesional, pero tampoco a su capacidad reproductiva concebida como parte de su ser mujer. Para dar cuenta de lo anterior analizamos cuatro entrevistas biográficas realizadas con la técnica de relatos de vida. Los resultados muestran las tensiones, conflictos y culpas que viven las mujeres profesionistas que trabajan y que son madres, así como la manera en que experimentan al mismo tiempo sensaciones de autoafirmación personal y sentimientos de orgullo.

Palabras clave: madres, maternidad, resignificaciones. 


\section{Women-mothers who work. The resignification of motherhood in female professionals in Guadalajara-Mexico}

\section{ABSTRACT}

The purpose of this article is to analyze the resignifications of motherhood among female professionals with children residing in Guadalajara, Mexico.

We argue that the transformations of the representation of motherhood and of the mother-like sociocultural institutions-are promoted and suffered by those women who "want everything", that is, those who do not give up their professional projects, and neither give up their reproductive capacities which they conceive as part of what being a woman means to them. To account for the above, we analyzed four biographical interviews conducted with the life story technique. The results show the tensions, conflicts and guilt experienced by the professional women who work and who are mothers, as well as the way in which they experience at the same time feelings of personal self-affirmation and feelings of pride.

Keywords: mother, motherhood, resignifications. 


\section{INTRODUCCIÓN}

En México, entre 2015 y 2016 el 49,8 por ciento de las personas que cursaban estudios profesionales eran mujeres (Secretaría de Educación Pública - SEP, 2015, citado en Instituto Nacional de Geografía y Estadística, 2017). Desde 2013, la tasa de egreso del nivel de la educación superior es mayor en las mujeres que en los hombres: está situada en un 21 por ciento frente a un 18 por ciento respectivamente (Centro de Investigación de la Mujer en la Alta Dirección, 2013).

Estas mujeres universitarias egresan de la Licenciatura aproximadamente a los veintiuno y veintidós años, lo que corresponde con la edad de inserción laboral de las mujeres mexicanas, que ocurre principalmente después de los veinte años (Medor, 2014). A esta edad, también, las mujeres mexicanas suelen tener su primer embarazo (veintiún años) según datos del INEGI, lo que permite fácilmente concluir que muchas de esas mujeres profesionistas se verán en la necesidad de compaginar la maternidad con el trabajo.

Es por esto que diversas autoras (Contreras y Castañeda, 2016; Manni, 2010; Palomar, 2009; Solé y Parella, 2004; Zicavo, 2013) aseguran que las mujeres madres y profesionistas experimentan tensiones, angustias o culpas al vivir simultáneamente las demandas ejercidas sobre sus cuerpos reproductivos y las exigencias de un sistema productivo capitalista que demanda éxito económico y trabajo intensivo.

Como se puede observar, el acceso a la educación superior, a la formación profesional, la posterior inserción en el mercado de trabajo y la construcción de trayectorias de vida donde trabajar no es una opción sino una elección de vida son sin duda factores que tienen consecuencias en el significado que se otorga a la maternidad. De acuerdo con Tania Sanhueza (2005), la maternidad se ha convertido en una posibilidad más del abanico de posibilidades del ser mujer, una posibilidad que puede o no ejercerse.

Coincidimos con Manni (2010) respecto de que las mujeres profesionistas reflejan quiebres y rupturas en cuanto a las prácticas y acciones asociadas 
tradicionalmente con el ser mujer. Asimismo, partimos del supuesto de que las mujeres que tienen cierta preparación cultural y educativa y que construyen un proyecto de vida propio son las que contribuyen de forma más significativa a la transformación del sentido de la maternidad y a la emergencia de maternidades menos intensivas o convencionales (Alberdi et al., 2000, citado en Solé y Parella, 2004; Manni, 2010).

Estudios como el de Zicavo (2013), titulado Dilemas de la maternidad en la actualidad: antiguos y nuevos mandatos en mujeres profesionales de la ciudad de Buenos Aires, afirman que en los sectores medios la vida profesional de las mujeres les ha permitido resignificar la maternidad, ya que ahora no se trata del único modo de realizarse y otorgar sentido a su existencia. El trabajo para las mujeres se ha convertido en una fuente de ingresos que, además, ofrece satisfacciones y éxitos. Entonces, aunque la maternidad continúe siendo un elemento clave para la constitución de la identidad femenina, ya no es el único ni el más importante referente. Ahora bien, la autora sugiere que en Buenos Aires, Argentina, para las mujeres de los sectores profesionales de clase media el dedicarse solo a la maternidad ya no otorga el mismo prestigio que en generaciones anteriores, por lo que estas mujeres están en medio de un nuevo mandato: el de ser madres que trabajan. Este mandato muchas veces es transmitido por los padres, parejas, amigos o amigas de las participantes, quienes reiteradamente les hacen hincapié en lo importante del trabajo y en que no deben abandonar su vida laboral por la maternidad.

Por su parte, el trabajo de Manni (2010) exhibe cómo algunas de las mujeres que participaron en su investigación señalan que su profesión no es un impedimento para llevar a cabo su maternidad. Sin embargo, la autora señala que las participantes de su investigación se caracterizan porque se casan y tienen hijos a mayor edad, o bien se incorporan al mercado de trabajo más tardíamente y permanecen en este por más años. Esto evidencia un esfuerzo de solución individual al problema de conciliar maternidad y trabajo, así como una postergación de una u otra actividad.

En la investigación de Manni se puede observar que las mujeres combinan roles más innovadores y flexibles con ideas más tradicionales acerca de la maternidad, lo que muestra que hay tensiones y contradicciones entre las prácticas y representaciones ideales de género y maternidad. Esta autora sostiene que en estas participantes las representaciones acerca de quiénes deben llevar a cabo el trabajo no remunerado han ido cambiando más rápidamente que las prácticas de cuidados y de atención del hogar, las cuales en su mayoría siguen a cargo de las mujeres. La solución que las mujeres participantes en el trabajo de Manni encuen- 
tran para resolver esto es recurrir a otras mujeres (nanas, sirvientas, madres), o bien orquestar la atención de los hijos a distancia.

Solé y Parella (2004) buscaron analizar los factores materiales e ideológicos que participan en la experiencia de maternidad de mujeres con actividades profesionales absorbentes. Las autoras encontraron que las prácticas de las mujeres profesionistas han cambiado, pues se muestra una mayor disposición horaria al trabajo, una búsqueda y permanencia en el trabajo no solo por necesidad económica sino porque este les resulta gratificante, les ofrece éxito profesional, les brinda la posibilidad de viajar o las dota de un elevado estatus social. El grupo de mujeres que participó en ese estudio no quería renunciar a la maternidad, ni a la familia, ni al trabajo. Sin embargo, las autoras observan que las representaciones e imaginarios de la maternidad intensiva siguen estando presentes en las mujeres, lo que las hace vivir con ciertas culpas. Pese a todo, su falta de tiempo implica que su maternidad se circunscriba a los fines de semana y consista en tiempos de ocio o diversión compartidos con los hijos. Este grupo de mujeres, además, asumen que la maternidad que llevan a cabo es difícil, por lo que dicen no considerarla peor o menos valiosa que la maternidad intensiva. Además, asumen que con ese tipo de maternidad están brindando buenos ejemplos para sus hijos.

Como se puede ver, estas mujeres viven entre prácticas y discursos que en ocasiones son contradictorios, ya que algunas veces experimentan culpa y hablan de que el ideal de maternidad es la tradicional. Sin embargo, en otras ocasiones expresan orgullo por mostrar nuevos modelos de maternidad a sus hijos y por la satisfacción que les brinda poder hacer ambas tareas.

En ese marco es que este documento tiene como objetivo el análisis del impacto que tiene el trabajo en la manera en que mujeres madres significan y resignifican la maternidad tradicional. El artículo está organizado de la siguiente manera. Luego de esta introducción, sigue una aproximación teórica a los conceptos de maternidad y significado. Luego, describimos cómo llevamos a cabo la investigación. Posteriormente, mostramos los principales hallazgos del estudio. Por último, reflexionamos en torno a los cambios y permanencias en el significado de la maternidad de mujeres profesionistas con hijos.

\section{SOBRE LAS MATERNIDADES Y LAS PRÁCTICAS (UN MARCO TEÓRICO CONCEPTUAL)}

Ser madres y vivir la experiencia de la maternidad «no se deriva de la función reproductiva de las mujeres, sino que es un proceso históricamente determinado 
y, como tal, sus características dependen de las relaciones sociales y de las elaboraciones culturales a través de las cuales las mujeres construyen su maternidad» (Sánchez-Bringas, 2003, p. 21). Es necesario incluir una serie de variables, como las condiciones socioeconómicas, las redes sociales, la historia reproductiva y las diferentes estructuras de significados que matizan la experiencia materna.

La maternidad, así entendida, no es «la esencia» de lo femenino, sino justamente un poderoso modelo cultural/ideológico que, si en el plano ideal/normativo no tiene mayores fisuras, al ser trasladado al plano psicológico y de las conductas presenta ambigüedades, conflictos y contradicciones (González, 1993). En palabras de Cristina Palomar:

La práctica de la maternidad parece sintetizar tanto las contradicciones como los ideales del género en nuestra sociedad, influyendo en la producción de una experiencia femenina, compuesta por automatismos, tradiciones, costumbres y prescripciones sobre lo que una mujer debe ser; como práctica real o como posibilidad, es algo que a toda mujer se le plantea en algún punto de su proceso vital, si bien que de diversas maneras. La maternidad se presenta de tal forma «naturalizada» como expresión del género, que se vive automáticamente, sin que medie un proceso reflexivo consciente que permita dar cuenta de los motivos que llevan a una mujer a tomar la decisión de tener hijos (Palomar, 2005, p. 54).

De acuerdo con Yanina Ávila, «la relación de las mujeres con la maternidad es un proceso tan naturalizado y mitificado que elegir no ejercerla, sobre todo de manera voluntaria, se convierte en un factor de tensión, que se expresa en la estigmatización y la presión social» (Ávila, 2005, p. 125). La maternidad, se constituye como un mandato cultural de género, relacionado estrechamente con los procesos de construcción de identidades (Ávila, 2005).

En general la teoría coincide en que la maternidad es una construcción sociocultural e histórica y, como tal, establece una serie de condicionamientos no solo en cuanto a su ejercicio, sino también en la propia aspiración de ser madre a cierta edad y, preferentemente, bajo ciertas condiciones (Badinter, 2010; Castilla, 2009; Miranda, 2000; Palomar, 2007, 2009; Castañeda-Rentería, 2019). Por lo tanto, la maternidad es un proceso complejo y lleno de ambigüedades que determina y configura la subjetividad de las mujeres, sean madres o no, por lo que amerita profundos análisis localizados (Palomar, 2009).

La vivencia de la maternidad es única en cada mujer y está determinada por múltiples aspectos. Las características educativas y laborales, así como la utilización de métodos anticonceptivos, son algunos de los aspectos que han transformado la forma en que las mujeres conciben y viven $-\mathrm{o}$ no- la maternidad. 
Un ejemplo es el trabajo de Hiroko Asakura, Cambios en significados de la maternidad: la emergencia de nuevas identidades femeninas, publicado en 2005 y cuyo objetivo era dar cuenta de los significados de la maternidad en la identidad femenina de mujeres de clase media urbana en la Ciudad de México. A través de entrevistas a profundidad con mujeres que decidieron ser madres solteras y otras que decidieron no tener hijos, la autora da cuenta de la construcción de una identidad distinta de la tradicionalmente asignada a las mujeres.

Haciendo alusión a Lipovetsky (1999), Asakura asegura que la «tercera mujer» en nuestro país es producto de la contribución de varios factores: «la legitimidad del trabajo y los estudios, la desinstitucionalización del matrimonio y la familia, la aparición de otro tipo de relación entre hombres y mujeres, el control de la fecundidad, etc.» (2005, p. 94) La pregunta que surge es, según la autora, «si los mitos de la maternidad ya no funcionan, si estas mujeres son realmente libres del peso del imaginario social» (Asakura, 2005, p. 94). La autora sostiene que no es así, y que estas mujeres buscan otras opciones alternativas de maternidad como el cuidado a los demás, parientes o animales, para reparar «su falta», pues estas identidades no han logrado legitimarse socialmente.

Lo encontrado por esta investigadora coincide con otras indagaciones internacionales que también han evidenciado la coexistencia de los modelos tradicionales y los que aparecen como nuevos y alternativos. Una de estas investigaciones es la de María Victoria Castilla (2009). La autora realizó un trabajo de tesis doctoral titulado Mujeres madres. Continuidades y cambios en los sentidos y experiencias de la maternidad argentina, donde se preguntó cuáles eran las características que asume la maternidad en el marco de los procesos de individualización en un contexto como el latinoamericano. El estudio se realizó en mujeres de clase media de la ciudad de La Plata, en Argentina.

Ella identifica una brecha entre los modelos tradicionales (maternidad intensiva, dedicación exclusiva al hogar) y las prácticas y experiencias propias de ser mujer, madre y vivir en familia, que explica de la siguiente manera:

[...] la brecha entre los modelos y prácticas/experiencias sería el producto de los cambios culturales en nuestra época, referentes a los crecientes procesos de individualización. Me refiero a los cambios rápidos y profundos en las maneras de vivir en familia y ser madre, sin que, al mismo tiempo, las madres hubieran reformulado las representaciones colectivas que se hacen de la maternidad y la vida en familia (Castilla, 2009, p. 9).

Lo que la autora encuentra es una diversidad de formas de practicar la maternidad que toman distancia de los «modelos ideacionales de la maternidad», debido 
a la situación histórica particular de las mujeres. La hipótesis que trabajó en su investigación hacía referencia justo a que «la brecha entre modelos y prácticas/ experiencias sería el producto de los cambios culturales de nuestra época, referentes a los crecientes procesos de individualización» (Castilla, 2009). Lo que observó fue la coexistencia entre los modelos ideacionales tradicionales de la maternidad, con nuevas - e incluso individualizadas - formas de ejercer y poner en práctica esos mismos modelos.

El trabajo de Sanhueza (2005) realizado en Chile se pregunta si la participación de la mujer de clase media en espacios distintos del doméstico ha disuelto en algún grado el ejercicio de la maternidad como elemento definitorio de la identidad femenina. La autora da cuenta de una continuidad/ruptura entre las generaciones encabezada por una mujer profesional con proyecto propio, donde la maternidad es una posibilidad más de la gama de metas en la vida de la mujer. Con un trabajo que incluyó el acercamiento con dos generaciones de mujeres — madre e hija -, identificó la diferencia entre el sentido de la maternidad para una y otra generación, y señala:

A diferencia de la generación de 1960 donde la maternidad se definía en términos de entrega, sacrificio, formación moral y complicidad, en la generación de 1990 la significación otorgada a la maternidad es desde la idea de la responsabilidad es esta noción la que hace no solo aplazar la llegada de les hijes [sic], sino pensar incluso en no tenerles (Sanhueza, 2005, p. 165).

A la pregunta de ¿cómo lo han logrado?, la autora identifica en el discurso de las propias madres la grieta que permite la resignificación del ser mujer: «a ti no te va a pasar lo que a mí». El trabajo asalariado, extradoméstico, sería el principal pilar para la construcción de esta nueva mujer, señalando que las mujeres que trabajan remuneradamente poseen una identidad dividida entre maternidad y trabajo.

Escindida, ¿por qué? ¿Trabajo y maternidad no se conjugan? Si consideramos que la identidad de une sujete [sic] se construye desde las prácticas y experiencias vitales y con base en el lugar que este ocupa en la sociedad, cómo incorporamos aquellas prácticas que no se encuentran en la lógica de «para y de los otros» (Sanhueza, 2005, p. 155).

La autora concluye su artículo con una idea, quizá provocadora: dice «las mujeres de los años noventa están más próximas en la construcción de sí mismas a estereotipos de un modelo masculino que al femenino tradicional, lo cual indica una tensión actual en las construcciones de género y las inconexiones en el legado» (Sanhueza, 2005, p. 186). 
Recapitulando, este trabajo se ubica en torno a la discusión que considera la maternidad como una construcción sociocultural, históricamente determinada, que se finca sobre el cuerpo de mujer y los supuestos de la existencia de una «esencia femenina», constituyéndose de esta forma en un elemento dominante de la configuración de las identidades y, en particular, de la constitución de la identidad de género, tanto de las mujeres madres como de las que no lo son. La maternidad como representación logra conjugar los atributos construidos como femeninos en nuestra sociedad: entrega, servicio, el ser para los otros.

La maternidad constituye una institución que norma y define de una manera importante la feminidad para mujeres madres y no madres. Al mismo tiempo, configura una experiencia de vida que implica decisiones, prácticas, actitudes que cualquier mujer experimentará como suyas en un momento de su vida. Vista desde aquí, la maternidad se vuelve la representación que condensa y expresa lo femenino, y al mismo tiempo un modelo inalcanzable para las mujeres que viven sus experiencias maternales de manera situada e interseccional.

En este marco se podría pensar que los significados atribuidos a la maternidad como representación, como modelo y como experiencia femenina se transforma en la misma proporción que las trayectorias femeninas se insertan en espacios laborales novedosos o ante la posibilidad de elegir simplemente no tener hijos. Sin embargo, trabajos como el de Castañeda (2019) y el de García y Castañeda (2018) evidencian cómo la maternidad en tanto núcleo de sentido femenino se mantiene pese a las posibilidades biográficas del ejercicio de una profesión, o del hecho de no tener hijos, en un proceso acumulativo que integra en la misma experiencia múltiples sentidos y significados en tensión, incluso contradictorios, que convierten la maternidad en un fenómeno mucho más complejo.

El objetivo de esta comunicación es identificar y analizar la manera en que mujeres posicionadas desde su trabajo profesional piensan, resignifican y otorgan sentido a sus maternidades. Para ello, entendemos el sentido en los términos planteados por Alonso (1998), es decir, siempre en contexto e inmerso en un conjunto de acontecimientos, y que responde a ciertos fines más amplios, propios de un sistema o de un nivel lógico superior. Conforme a Reguillo (2000), los actores, desde su posición, elaboran un relato de un tema. Si bien el relato no siempre es consistente, sí que habla de anclajes identitarios y de formaciones discursivas. Justo por lo anterior, se dice que el sentido tiene un carácter extralingüístico, está social e históricamente situado (Reguillo, 2000). Se puede apreciar cierta coincidencia entre Alonso (1998) y Reguillo (2000) en que el sujeto está enmarcado en un cuerpo social, por lo que es producto y formador de lo social. 


\section{METODOLOGÍA}

El enfoque fue cualitativo y el diseño de investigación fue biográfico narrativo. La recolección de información se llevó a cabo a través de entrevistas semiestructuradas y relatos de vida. El muestreo fue por conveniencia. Para este trabajo se eligieron los relatos de cuatro mujeres profesionistas de 38 a 44 años con hijos o hijas residentes en Guadalajara, México. En todos los casos se usaron los pseudónimos elegidos por las mismas participantes: Estela, Pancha, Nancy y Valeria.

En la tabla 1 se muestran algunos datos sobre las mujeres profesionistas madres en relación con su edad, estado civil, hijos o hijas, grado de estudio y trabajo actual.

Tabla 1. Generales de las informantes profesionistas con hijos

\begin{tabular}{|c|c|c|c|c|c|}
\hline & Edad & Estado civil & Hijos o hijas & $\begin{array}{l}\text { Nivel de } \\
\text { estudios }\end{array}$ & Trabajo actual \\
\hline Estela & 38 & $\begin{array}{l}\text { Casada civil y } \\
\text { religiosamente }\end{array}$ & $\begin{array}{l}\text { Hombres } 10 \text { y } 4 \\
\text { años }\end{array}$ & $\begin{array}{l}\text { Doctora en } \\
\text { Ciencias } \\
\text { Sociales }\end{array}$ & $\begin{array}{l}\text { Administrativa } \\
\text { en institución } \\
\text { educativa }\end{array}$ \\
\hline Nancy & 41 & Casada civil & $\begin{array}{l}\text { Mujer } 15 \text { años } \\
\text { Hombre } 12 \text { años }\end{array}$ & $\begin{array}{l}\text { Maestra en } \\
\text { Pedagogía }\end{array}$ & $\begin{array}{l}\text { Maestra en } \\
\text { preescolar }\end{array}$ \\
\hline Pancha & 41 & $\begin{array}{l}\text { Divorciada } \\
\text { Actualmente } \\
\text { unida }\end{array}$ & $\begin{array}{l}\text { Mujer } 16 \text { años } \\
\text { Hombre } 8 \text { años }\end{array}$ & $\begin{array}{l}\text { Doctora en } \\
\text { Estudios } \\
\text { Urbanos }\end{array}$ & $\begin{array}{l}\text { Directora } \\
\text { de área en } \\
\text { institución } \\
\text { gubernamental }\end{array}$ \\
\hline Valeria & 38 & Comprometida & Hombre 8 años & $\begin{array}{l}\text { Licenciada en } \\
\text { Comunicación }\end{array}$ & $\begin{array}{l}\text { Fotoperiodista } \\
\text { free lance }\end{array}$ \\
\hline
\end{tabular}

Elaboración propia a partir de la información obtenida de las entrevistas.

\section{HALLAZGOS}

Los resultados se organizaron en dos subtemas que a continuación se presentan:

\section{El trabajo en la vida de las mujeres profesionistas con hijos}

Los casos de tres de estas cuatro profesionistas con hijos se caracterizan por trayectorias tanto académicas como laborales marcadas por la idea de «ser alguien» que promovieron tanto los padres como las madres, con excepción del caso de Estela, cuya madre le prohibió estudiar la preparatoria y al cual volveremos más adelante. 
Para Nancy, Pancha y Valeria, estudiar la universidad fue algo natural: «era lo que seguía». Ambos padres las apoyaron, y aunque no necesitaban hacerlo, se integraron a diferentes espacios laborales aún antes de egresar de sus carreras. Nancy, por ejemplo, relata cómo desde los ocho años encontraba la forma de obtener ingresos vendiendo limones de un árbol que su abuela tenía en casa. Ya en la secundaria trabajaba en una tienda de ropa. Todo lo hacía a escondidas de su padre y bajo la protección de su madre, pues según recuerda, a su papá le preocupaba que ganar dinero la distrajera de sus estudios.

El caso de Estela es diferente. Su madre, ama de casa, consideraba que estudiar no era bueno para sus hijas, pues decía que «las mujeres que estudian no se casan». Su padre no pensaba igual. Así que cuando Estela terminó la secundaria, para evitar un enfrentamiento entre sus padres, decidió quedarse en casa. Cuando alcanzó la mayoría de edad, decidió seguir estudiando con el apoyo de su hermana mayor y el beneplácito de su padre. Estela es la primera generación universitaria de su familia y cuenta con estudios doctorales. Al igual que los otros casos, ingresó a trabajar antes de egresar de la licenciatura.

Como se puede apreciar, las tres primeras participantes crecieron escuchando de sus hermanos y padres lo importante de estudiar y posteriormente trabajar, por lo que podríamos decir que, tal como afirma Zicavo (2013), crecieron y han sido socializadas con el mandato de ser mujeres que estudian y trabajan.

No obstante, ninguna de estas mujeres tuvo duda de que alguna vez sería madre. Nancy, por ejemplo, eligió la carrera de educadora justo por la compatibilidad que el ejercicio profesional tenía con el cuidado y administración de un hogar, así como la posibilidad de atender «como se debe» al esposo y a los hijos. En los otros casos, había la idea de que en su momento tendrían que implementar alguna estrategia que les permitiera ser profesionistas y trabajar aun teniendo hijos: «Así como fui a estudiar, era para mí normal ser mamá» (Pancha).

Tanto Valeria como Pancha tienen hijos de relaciones anteriores a las actuales. Ambas relatan como normal el proceso de encontrar una pareja, cierta estabilidad con ella, unirse (con o sin matrimonio) y tener hijos. Ninguna de ellas pensó en renunciar a su trabajo cuando nació su primer hijo: «el trabajo era parte normal en mi vida y una parte necesaria para mantener o alcanzar un estilo de vida» (Pancha). En el caso de Valeria, se separó del padre de su hijo al mes de nacido este: «No regresar, además, no fue opción, ahora tenía que arreglármelas con un bebé».

Nancy se unió con su actual pareja convencida de que quería hijos y en una situación donde había planeado desde la elección de carrera que su trabajo fuera compatible con su actividad como mamá. Por su parte, Estela comenta que nunca 
consideró necesario dejar de trabajar, aunque tuvo a su primer hijo egresando de la licenciatura, y después de la maestría, al segundo; es decir, programó sus embarazos en relación con sus proyectos de estudio y laboral.

Los relatos de Nancy y de Estela coinciden con el esfuerzo racional e individual que Manni (2010) sugiere que hacen las mujeres profesionistas para compatibilizar su trabajo con su maternidad. Solo que, a diferencia de las participantes de Manni (2010), el esfuerzo de Estela y Nancy no consistió en posponer la maternidad o el ingreso al trabajo, sino desde la elección de carrera, planificar la compatibilidad de ambas actividades, o bien programar racionalmente los tiempos de embarazo y estudio.

Algunas de las participantes no han logrado compatibilizar tan armónicamente trabajo y maternidad como Estela y Nancy. Por ejemplo, Valeria, quien tiene una profesión que podríamos denominar «masculinizada», pero una vez que nació su hijo, todo su proyecto profesional y laboral se vio frustrado. Recuerda que alguna vez recibió una llamada de su jefe a las diez de la noche para que fuera a tomar unas fotografías a una reunión en la que se encontraban algunos diputados. «Le dije: "no puedo, el niño está dormido”, y me contestó: “¡Déjalo! Toma las fotos y te regresas"». Muy a su pesar, pero con la responsabilidad de un hijo, Valeria decidió renunciar y trabajar por su cuenta, lo cual implica trabajo, sí, pero incertidumbre sobre fechas de pago, por ejemplo.

Una situación parecida vivió Estela, quien se ha visto cuestionada en tanto su compromiso con el trabajo o con sus estudios cuando menciona a sus hijos:

También es un choque para mí que me digan que perdí tiempo con la maternidad; yo digo, bueno es que yo tengo que combinar dos cosas y no puedo ir a la velocidad que otros pueden cuando no tienen hijos. Yo siempre tuve muy claro que no quería ser una académica sin hijos, lo vi en el caso de una amiga y me cayó el veinte, yo no quiero una vida así, yo no quiero sacrificar esa parte de mí (Estela).

Valeria es la única que se ha preguntado si estuvo bien o no tener un hijo. Este cuestionamiento lo hace sabiendo que su proyecto laboral se vino abajo a partir del nacimiento de su niño, situación que, según ella concibe, no necesariamente sería distinta si contara con el apoyo de una pareja. Según Valeria, no se trata de que sea madre soltera, se trata simplemente de que la maternidad es incompatible con su profesión. Y agrega con un tono burlón: «No, no lo he superado y no lo voy a superar. ¡Veme la cara bien jodida!... Además, se acercan los cuarenta y estoy en crisis, necesito pago por quincena, necesito producir». 
A pesar de lo anterior, el trabajo para estas cuatro mujeres representa, más que una necesidad económica, una parte fundamental para definirse y constituirse como sujetos. Es claro el vínculo que estas mujeres construyen entre «realización personal» $\mathrm{y}$ «proyecto laboral». También es claro que la «realización personal», para las participantes, parece no tener nada que ver con «realizarse como mamá».

El proyecto respecto de los hijos resulta importante y trascendente para ellas como madres; sin embargo, su realización personal no necesariamente pasa solo por ahí. La mujer que tiene hijos no es devorada por la madre de esos hijos, sino que está en constante pugna por no desaparecer como sujeto. El ancla para que esto no suceda es el trabajo profesional, que es visto como algo propio, como una ganancia personal, en palabras de Nancy «es como un balance para mi ser, entre lo que quiero y lo que puedo». Valeria, por ejemplo, recrimina a algunas amigas que después de estudiar y trabajar ahora se dedican a sus hijos. «¿Por qué te dejaste? ¿Por qué terminaste siguiendo el sueño de otro? [...] Yo me doy una, cuatro veces en el hocico, pero me levanto y sigo, yo, por mí». Entonces pareciera que, tal como propone Zicavo (2013), el trabajo ha permitido a las mujeres contar con formas de realización distintas de la maternidad.

Ahora bien, la forma en que las participantes de nuestro estudio dan sentido a su trabajo cambia cuando aparecen los hijos. Se argumenta una preocupación e interés por su bienestar en términos de una buena educación —entiéndase buena escuela-, clases extraescolares, vacaciones, ropa, diversión, y la manera en que con su trabajo contribuyen o pagan en su totalidad esos gastos. Conviene señalar que el trabajo que desempeñan, además de la posibilidad de pagar gastos familiares, les permite sentirse productivas (Valeria), satisfechas (Pancha) o reconocidas (Nancy). Esto concuerda con lo que sostienen diversas autoras (Solé y Parella, 2004; Zicavo, 2013), quienes señalan que el trabajo no es solo una forma de hacer frente a requerimientos económicos, sino que se ha convertido una tarea que proporciona gratificación, satisfacciones y éxitos para las mujeres profesionistas.

Sentirse así (satisfechas, con un proyecto propio), según dicen, les permite también ser mejores mamás. Lo anterior no significa que se consideren «buenas madres», pero desde su situación consideran que el trabajo que realizan compensa el desgaste que ocasiona el cuidado de los otros. Les permite, además, pensarse a sí mismas como ejemplos de superación y trabajo para sus hijos, pero sobre todo para sus hijas. A la pregunta de si dejarían su trabajo, Pancha responde: «No, no. Yo me siento satisfecha con lo que hago y estoy segura que eso tiene que retribuir en algo bueno para ellos». 
Estas mujeres saben que no son «buenas mamás» en términos de entrega y dedicación absoluta a sus hijos e hijas, pero defienden el tipo de madres que pueden ser, construyen otras maternidades donde la importancia de que los hijos e hijas sean independientes, autónomos, esforzados y «piensen y luchen por su desarrollo personal» (Pancha) es prioritaria a la dedicación exclusiva. Estela siempre les recuerda a sus hijos que «ellos se van a ir y no tienen por qué dejar de hacer su vida por su mami, deben pensar en su vida». Lo anterior no quiere decir que no sientan culpas, miedos y estrés.

Esta manera de pensar su maternidad como un ejemplo para sus hijos e hijas ha sido encontrada en trabajos previos (Solé y Parella, 2004) y explicada como una forma de validar y reconocer los esfuerzos y dificultades que comportan maternidades que podrían considerarse como no convencionales o no intensivas. Dicha resignificación de la maternidad, tanto en nuestras participantes como en el trabajo de Solé y Parella (2004), no está exenta de culpas, tal como veremos en la siguiente sección.

\section{Ser mamá: la tensión entre las culpas y el orgullo de poder con todo}

Tal como mencionamos en la sección anterior, ninguna de las cuatro mujeres cuestionó que en su futuro serían madres. Tampoco han considerado siquiera dejar de trabajar para dedicarse por completo al cuidado de sus hijos o hijas, y confían que el tipo de maternidad que ejercen, aunque alejado del modelo ideal, es el mejor, porque permite que sus hijos e hijas tengan un determinado estilo de vida y acceso a recursos necesarios para el bienestar de estos, además de ofrecerles un ejemplo.

Pese a lo anterior, su vida no está libre de sentimientos de culpa o deuda. Por ejemplo, Pancha considera que ha quedado a deber tiempo y atención a su hija mayor. Sonríe cuando a sus cuarenta años recuerda que a sus veintitrés ella pensaba que el trabajo sería un rol adicional a su papel de madre: «La parte laboral ha sido siempre muy padre, pero esa parte de no estar cercano a lo que necesitan yo creo que sí pega» (Pancha). Nadie nunca ha recriminado a Pancha su papel como madre - y padre-; sin embargo, asume que no ha sido una madre en los términos que «una buena madre» debe ser, y dice: «La maternidad no me define como mujer, porque aunque es una parte muy relevante, creo que no me siento tan mamá, tan mamá, no me atrevo a definirme así» (Pancha).

Por su parte, Estela sí ha recibido recriminación sobre su papel como mamá por su propia madre. La relación entre Estela y su mamá está siempre en una 
constante tensión. Asegura que su mamá sabe que trabajar ya no es opción en una realidad económica como la que se vive actualmente: lo que la molesta de sobremanera es que siga estudiando,

Recuerdo mi primer viaje al extranjero. No le dije nada hasta que ya casi me iba para que no me estresara con eso de «¿cómo que vas a dejar a los niños?». Porque luego sí consigue hacerme sentir mal. Y sí, sí siento como esa culpabilidad porque te la hacen sentir. Mi esposo no, pero mi mamá sí. Una vez me dijo: «El niño te extrañó mucho y se llenó de sarpullido por estos días que no te vio» (Estela).

Por otro lado, Nancy se percibe un tanto incómoda cuando, al preguntarle sobre qué tipo de mamá es, cae en la cuenta que se parece mucho a su madre: «Soy una madre entregada, abnegada, comida lista, súper protectora, soy toda esa parte que no me gustaba de mi mamá [risas]. Pero a diferencia de mi mamá yo soy exigente, exijo calificaciones, platico con ellos, con las maestras. Esta última parte me gusta más [risas]».

Estas mujeres profesionistas con hijos dan cuenta de la existencia de un modelo ideal de madre que decidieron no seguir. Esto genera culpas y sentimientos de deuda con respecto a los hijos e hijas. Dichas emociones y sentimientos nunca llegan a ser lo suficientemente poderosos para hacerlas renunciar a su proyecto laboral. Ante esto, han encontrado una manera de resignificar ese ejercicio alternativo de maternidad: en el discurso de estas mujeres es posible encontrar argumentos que sostienen que pueden ofrecer otras cosas a sus hijos e hijas con las cuales podrían compensar, de algún modo, su no ser buenas madres en términos tradicionales.

Esto nos muestra que, tal como han sostenido otros estudios (Manni, 2010; Solé y Parella, 2004; García y Castañeda-Rentería, 2018), las prácticas y representaciones vinculadas con la maternidad no cambian al mismo ritmo. En estos casos, también observamos que, si bien las prácticas de las mujeres madres profesionistas están cambiando, la representación de la maternidad intensiva sigue arraigada fuertemente en el imaginario de las mujeres, lo cual genera culpas, incomodidades y autosanciones.

Además de esto, identificamos que para estas mujeres es un orgullo «poder con las dos cosas». Se saben criticadas, juzgadas, pero también están en una constante lucha por demostrar en estos dos ámbitos de su vida que pueden hacer las cosas. Estela lo vive constantemente: ella sabe que en su trabajo, lleno de «solteronas» y hombres, no es fácil que le den permiso de faltar por enfermedad 
de uno de sus hijos o para asistir a un festival por el Día de la Madre. La última vez que pidió licencia para cubrir sus estudios doctorales, la encargada le dijo: «Lo bueno es que no tienes hijos». Llevar a cabo sus dos roles es una batalla diaria para demostrar que se puede, demostrarlo en su trabajo, a su madre, y quizá a ella misma.

Por último, pensamos necesario resaltar que, con excepción de Valeria, las otras tres mujeres cuentan con la existencia de una red familiar que les posibilita desarrollar ambos papeles sin tanta culpa. Es notorio, por ejemplo, cómo el no haber «dejado» a sus hijos en guarderías es relatado como una muestra de que no son tan malas madres. Identificamos pues que, tal como se ha documentado previamente (Manni, 2010), una manera de paliar las tensiones y contradicciones que se generan entre la crianza de los hijos y el trabajo es recurrir a una red de apoyo solidario, principalmente femenino (madres, abuelas, nanas).

\section{CONSIDERACIONES FINALES}

Una de las características que comparten las informantes que aquí presentamos es su alta capacidad reflexiva y racionalidad. Planifican y organizan estratégicamente desde la elección de la carrera hasta los tiempos de embarazo. Con ello se puede ver que, en la época contemporánea, la elección de cuándo, cuántos y en qué circunstancias tener hijos es una posibilidad que marca y orienta la planificación de la propia vida, tal como han sostenido Beck y Beck-Gersheim (2002)

Las mujeres profesionistas con hijos tienen como eje de vida, más que la conciliación, la no renuncia. Se trata de mujeres que, si bien declaran que sus hijos son la prioridad de sus vidas, su maternidad no implica dedicar su vida por completo a ellos o ellas, sino que, entonces, la lucha por el ingreso económico - $\mathrm{y}$ en general la vida profesional - se resignifica $\mathrm{y}$, aunque sigue siendo un asunto de satisfacción y desarrollo personal, se declara como necesaria para dar lo mejor a los hijos. Sugerimos, además, que el trabajo profesional se vuelve un contrapeso necesario para la autoafirmación que hace posible resistir el anonimato con el que se realizan labores de cuidado y la desolación del ser para los otros.

Estas mujeres siempre están ideando estrategias para el cuidado de los hijos, la calidad del tiempo dedicado a ellos, las múltiples clases extraescolares, etc. Hacen lo posible por cumplir de la mejor manera con el ideal de ser una buena madre y lo hacen conscientes de que las realidades económicas y sociales son distintas de las de sus madres. Utilizan el argumento de la maternidad y el bienestar del niño (que asiste a clases extraescolares, se aproxima a un determinado 
consumo cultural y educativo, etc.) para mantener y explicar las actividades productivas y el trabajo. A la vez, se obtienen ventajas como el reconocimiento o la satisfacción. En suma, la práctica cambia, pero las razones son las mismas: el pensar en dar lo mejor al «otro», en este caso al hijo o hija.

Lo que no podemos dejar de lado es que estas mujeres han podido «hacer» de mamás en parte porque tienen trabajos «feminizados»: educadoras, etc. Sin embargo, en el caso de Valeria, quien es fotoperiodista, la manera de articular esto no es tan armoniosa o sencilla, lo que genera que las mujeres tengan que buscar nuevas estrategias y formas para ganarse la vida. Las mujeres que se han insertado a trabajos masculinizados, como en este caso, y que en estos encuentran dificultades para compatibilizar la vida profesional y la maternidad, terminan por preguntarse si la maternidad o el momento en que se eligió fue óptimo.

Desde su posición como mujeres que trabajan, estas madres argumentan que trabajar les permite ser mejores madres que si no trabajaran. No se trata de ser una «buena madre», sino que el trabajo les permite aprehender una dimensión propia para la felicidad y el desarrollo, «soportar» la entrega, el sacrificio y el anonimato de los cuidados y, finalmente, resignificar el ejemplo que dan como madres, pensándolo más allá de la entrega como un ejemplo de esfuerzo y trabajo.

Lo anterior no las exime de experimentar culpa y malestar por no responder al modelo tradicional de maternidad: también sienten que no son «tan mamás» justamente por no cumplimentar los requerimientos y lógicas asociadas con la maternidad hegemónica. Pero siempre encuentran formas de evadirlo.

Pese a esta culpa o malestar, estas mujeres no se cuestionan si es pertinente seguir o interrumpir el trabajo; es decir, tienen claro y defienden firmemente que quieren y desean seguir trabajando, lo cual muestra que el trabajo en la biografía y subjetividad de estas mujeres ocupa un lugar central y no negociable o renunciable.

Algunas mujeres, pese a trabajar, siguen desarrollando una maternidad entregada, intensiva, y en general, todas mantienen esa idea del maternaje como la posibilidad válida y deseable para ser madre. Esta sensación se aminora cuando hay redes de apoyo familiares que permiten ofrecer la afectividad y cuidados a los hijos de los que ellas no son capaces, pues la asistencia a guarderías y recursos de ese tipo no genera igual bienestar que lo anterior. 


\section{REFERENCIAS}

Alonso, Luis Enrique (1998). La mirada cualitativa en sociología. Introducción y Epílogo (pp. 15-33 y pp. 221-242). Madrid: Fundamentos.

Asakura, Hiroko (2005). Cambios en significados de la maternidad: emergencia de nuevas identidades femeninas. En M. Torres (ed.), Nuevas maternidades y derechos reproductivos (pp. 61-98). México, D. F.: El Colegio de México.

Ávila González, Yanina (2005). Mujeres frente a los espejos de la maternidad: las que eligen no ser madres. Desacatos. Revista de Antropología Social, 107-125.

Badinter, Elisabeth (2010). La mujer y la madre. Barcelona: La Esfera de los Libros.

Beck, Ulrich y Elizabeth Beck-Gernsheim (2002). La individualización: el individualismo institucionalizado y sus consecuencias sociales y políticas. Barcelona: Paidós.

Castañeda-Rentería, Liliana (2019). Mujeres profesionistas sin hijos: la defensa del modelo tradicional de maternidad desde la no maternidad. Desacatos, 60, 134-149.

Castilla Conles, María (2009). Continuidades y cambios en los sentidos y experiencias de la maternidad argentina. México, D. F.: Ciesas.

Contreras, Karla y Liliana Castañeda-Rentería (2016). Tensiones entre el cuerpo productivo de la mujer y la normatividad de género en torno a la maternidad. Revista Latinoamericana sobre cuerpos, emociones y sociedad, 21, 10-24.

Centro de Investigación de la Mujer en la Alta Dirección (2013). Estadisticas sobre mujeres empresarias en México. Recuperado de http://ipade.mx/wp-content/ uploads/2017/04/Estadisticas_sobre_mujeres_y_empresarias_en_Mexico.pdf

García, Diana y Liliana Castañeda (2018). La representación social sobre la maternidad $\mathrm{y}$ «el ser madre» en tres generaciones de mujeres: abuelas, madres e hijas. En Oresta López, María Luisa Martínez y Esperanza Tuñón (coords.), X. Estudios de Género: feminismos y temas emergentes en las ciencias sociales y la agenda nacional. Reflexiones y propuestas desde las ciencias sociales (pp. 759-788). México, D. F.: COMECSO, El Colegio de San Luis, CONACYT.

González Montes, Soledad (1993). Hacia una antropología de las relaciones de género en América Latina. México, D. F.: El Colegio de México.

Instituto Nacional de Geografía y Estadística - INEGI (2017). Estadísticas a propósito del Día Internacional de la Mujer. Datos nacionales. Recuperado de http://www. inegi.org.mx/saladeprensa/aproposito/2017/mujer2017_Nal.pdf [disponible en la red el 11 de febrero de 2018]. 
Instituto Nacional de Geografía y Estadística - INEGI (10 de agosto de 2015). Estadísticas a propósito del Día Internacional de la Juventud (12 de agosto). Consultado en: $\quad$ http://www.inegi.org.mx/saladeprensa/aproposito/2015/juventud0.pdf [disponible en la red el 12 de febrero de 2018].

Lipovetsky, Gilles (2012 [1999]). La tercera mujer. México, D. F.: Anagrama.

Manni, Luciana (2010). Cambios en las representaciones sociales e identidades genéricas de mujeres profesionales. La Aljaba Segunda Época, 14, 135-156.

Medor Bertho, Ducange (2014). Maternidad, jefatura de hogar y trabajo remunerado. Estrategias conciliatorias de un grupo de jefas de hogar en la Zona Metropolitana. En L. Castañeda (ed.), Mujeres, experiencias y retos. Guadalajara: Universidad de Guadalajara.

Miranda Guerrero, Roberto (2000). La vida al filo de las espadas. Familia, matrimonio, sexualidad y elección de pareja en el Obispado de Guadalajara. Guadalajara: CIESAS Occidente.

Palomar Verea, Cristina (2005). Maternidad: historia y cultura. Revista de Estudios de Género. La Ventana, 35-67.

Palomar Verea, Cristina (2007). Maternidad en prisión. Guadalajara: Universidad de Guadalajara.

Palomar Verea, Cristina (2009). Maternidad y mundo académico. Altheridades, 19(38), 55-73.

Reguillo, Rossana (2000). Anclajes y mediaciones del sentido. Investigación cualitativa en salud, dossier de la Revista de la Universidad de Guadalajara, enero. Guadalajara: Universidad de Guadalajara.

Sánchez-Bringas, Ángeles (2003). Mujeres, maternidad y cambio. Prácticas reproductivas y experiencias maternas en la Ciudad de México. México, D. F.: UAM Xochimilco, UNAM.

Sanhueza Morales, Tatiana (2005). De prácticas y significancias en la maternidad, transformaciones en identidad de género en América Latina. La Ventana, 146-188.

Solé, Carlota y Sonia Parella (2004). Nuevas expresiones de la maternidad. Las madres con carreras profesionales «exitosas». RES, 4, 67-92.

Zicavo, Eugenia (2013). Dilemas de la maternidad en la actualidad: antiguos y nuevos mandatos en mujeres profesionales de la ciudad de Buenos Aires. La Ventana, $38,50-87$. 